Research Article

\title{
Evaluation of Actin-1 Expression in Wild Caught Wuchereria bancrofti-Infected Mosquito Vectors
}

\author{
Moses Edache Entonu, ${ }^{1,2}$ Aliyu Muhammad (i), ${ }^{1,2}$ and Iliya S. Ndams ${ }^{2,3}$ \\ ${ }^{1}$ Department of Biochemistry, Ahmadu Bello University, Zaria, Kaduna State, Nigeria \\ ${ }^{2}$ Africa Centre of Excellence for Neglected Tropical Diseases and Forensic Biotechnology, Ahmadu Bello University, \\ Zaria, Nigeria \\ ${ }^{3}$ Department of Zoology, Ahmadu Bello University, Zaria, Kaduna State, Nigeria
}

Correspondence should be addressed to Aliyu Muhammad; amachida31@gmail.com

Received 20 November 2019; Revised 18 March 2020; Accepted 30 March 2020; Published 1 October 2020

Academic Editor: Giovanna Franciosa

Copyright ( 2020 Moses Edache Entonu et al. This is an open access article distributed under the Creative Commons Attribution License, which permits unrestricted use, distribution, and reproduction in any medium, provided the original work is properly cited.

\begin{abstract}
Background. Wuchereria bancrofti is the major cause of lymphatic filariasis transmitted by mosquito vectors. In the vector-parasite interaction and among other proteins, actin-1 has been implicated for successful transmission of the pathogen in laboratorycontrolled experiments. However, validation of this finding from the pathogen's natural environment is required. Objective. This study is aimed at evaluating actin-1 expression upon Wuchereria bancrofti infection in mosquito vectors collected during an epidemiology study in Tsafe Local Government Area of Zamfara State, Nigeria. Methods. Mosquitoes were collected and identified using morphological keys, which include length of maxillary palps, pale spots on the wings, and scale patterns on the abdomen. This was followed by detection of the $188 \mathrm{bp} \mathrm{SspI} \mathrm{marker} \mathrm{of} \mathrm{Wuchereria} \mathrm{bancrofti} \mathrm{infection} \mathrm{using} \mathrm{polymerase} \mathrm{chain} \mathrm{reaction}$ (PCR). The mRNA levels of the actin-1 gene were evaluated in the infected Anopheles gambiae sl and Culex quinquefasciatus and their controls, which were adult reared from the larvae in the study area. Results. The mosquitoes were identified to be Anopheles gambiae sl and Culex quinquefasciatus, while infection by Wuchereria bancrofti was confirmed by amplification of the $188 \mathrm{bp} S s p I$ marker. A 4.85 and 4.09 relative fold increase in actin-1 gene expression in Wuchereria bancrofti-infected Anopheles gambiae sl and Culex quinquefasciatus was observed. Thus, for the first time we reported that the actin-1 gene in wild caught mosquito vectors (Anopheles gambiae sl and Culex quinquefasciatus) infected with Wuchereria bancrofti is upregulated. Conclusion. The actin-1 gene is upregulated and similarly expressed during $W$. bancrofti infection in mosquito vectors in the study area and this may likely serve as a biomarker and viable strategy for the control of parasite transmission in endemic areas.
\end{abstract}

\section{Introduction}

Lymphatic filariasis is a neglected tropical disease (NTD) implicated in the blocking of the lymphatic system caused by parasitic helminths [1]. There are about 500 species of filariae known to infect vertebrates, out of which only eight main species infect humans. These include Wuchereria bancrofti, Brugia malayi, Brugia timori, Onchocerca volvulus, Loa loa, Mansonella perstans, Mansonella streptocerca, and Mansonella ozzardi. The preferred predilection sites for these parasites are the lymphatic vessels and lymph nodes where they induce development of disfiguring and debilitating clinical symptoms [2]. It has been reported that 886 million people of different countries are living within areas that require preventive chemotherapy to stop the spread of infection. Approximately $80 \%$ of these people are living in the following 10 countries: Angola, Cameroon, Côte d'Ivoire, Democratic Republic of the Congo, India, Indonesia, Mozambique, Myanmar, the United Republic of Tanzania, and Nigeria [3]. Fascinatingly, the burden of lymphatic filariasis in Nigeria is predominantly caused by Wuchereria bancrofti [4].

The geographical locations and habitat types are major influences where mosquito species function as vectors in any particular endemic area [5]. Current research studies are now focusing on expanding new strategies to abate 
vector-mediated pathogen transmission which has extended gradually to increasing priority for research on the mosquito-filarial system. However, the success of mosquito-borne disease control is often challenged by reintroduction and resurgence emanating from new or residual human infections that are further disseminated by the vectors, especially when effective human treatment is inadequate. Although the application of insecticides has been largely to reinforce mosquito control for decades, toxicity to humans and emergence of insecticide-resistant traits among mosquito populations have been of great concern, thus reducing the effectiveness of other control measures [6].

Mosquitoes are often exposed to pathogens during feeding through breaks in their cuticle following pathogendriven cuticular degradation. To resist this, they mount innate cellular and humoral immune responses which sometimes lead to the death of the pathogen through different mechanisms such as lysis, melanization, and hemocyte-mediated phagocytosis [7]. Mosquitoes' immunological, physiological, and structural components can prevent and ameliorate the establishment of filarial parasites [7, 8]. Migratory timing and mechanical crossing of vector midgut barriers [7], as well as ingestion of mitochondria of the thoracic muscle cells of the mosquito, constitute parts of the physical strategies employed by Wuchereria bancrofti in order to adapt within the mosquito environment $[9,10]$. Epithelial cells of the peritrophic matrix in the mosquito midgut are the first line of defense against many pathogens acquired during blood feeding, and these cells can synthesize several antimicrobial peptides (AMPs). The transcription of innate immunity genes encoding for AMPs is highly dependent on several signalling cascade pathways, including the Janus kinase-signal transducer and activator of transcription (JAK-STAT), toll-like receptor, and immune deficiency pathways [11]. The humoral defense proteins of $C x$. quinquefasciatus have been elucidated in a laboratory-controlled experiment, during which five proteins were upregulated with a molecular weight of $66,22,14,7$, and $40 \mathrm{kDa}$ for transferrin, attacin, lysozyme, defensin, and actin, respectively [12], of which actin which was referred to actin-1 during the primer design [13] is the most critical to parasite infection and development. These proteins are part of the vectors' immune complexes that evolved in response to their interactions with lifecycle stages of pathogens and function in a variety of intracellular processes aimed at ameliorating the cost of vectorial capacity [14]. However, mosquito infected with Wuchereria bancrofti from its natural environment has not been examined for these proteins of which actin-1 is the most abundant and often involved in the parasite-vector interaction [15]. Actin-1 is part of the mosquito gut tissue involved in an antagonistic function of limiting parasite infection [16]. Evaluating actin-1 expression in an infected mosquito vector could serve as a possible biomarker for vaccine and drug development in the new strategy for the control of the parasite transmission in endemic areas. This study is aimed at evaluating actin-1 expression in wild caught Wuchereria bancrofti-infected mosquito vectors in Tsafe Local Government of Zamfara State, Nigeria.

\section{Materials and Methods}

2.1. Chemicals and Reagents. TRIzol reagent, isopropyl alcohol, chloroform, and ethanol (absolute) were obtained from Sigma-Aldrich (USA), RNase-free water, SDS, primers, and agarose powder from Inqaba Biotech South Africa, dNTPs and Taq polymerase from Thermo Fisher Scientific (USA), First-strand cDNA synthesis kit and Luna Universal qPCR master mix from New England BioLab, and ethidium bromide and RNAlater were obtained from Thermo Fisher Scientific (USA). All other reagents were of analytical and molecular grade. Strict adherence to manufacturer's instruction was maintained.

2.2. Study Area. The study area was Tsafe Local Government Area in Zamfara State Nigeria with the coordinate $11.57^{\prime} \mathrm{N}$ and $6.55^{\prime} \mathrm{E}$ which is about fifty (50) kilometre from Gusau, the state capital. The ethnic groups in the area are predominantly Hausa, Fulani, and Bare-Bare. The major agricultural activity is farming, rearing of animals, and trade [17].

2.3. Sample Collection. Convenient method of sampling was used to randomly select three wards from the Local Government Area. In each ward, two sites were selected and five houses were subsequently chosen in which both infected and control mosquitoes were collected by pyrethrum knockdown (PKD) using Baygon as an insecticide of choice. This was used in spraying the indoor-resting mosquitoes between 06:00 am and 10:00 am [3, 18]. The collected mosquitoes were immediately stored in RNAlater at $-20^{\circ} \mathrm{C}$. 100 mosquito larvae were also collected from the same population to be used as controls. These larvae were identified to be Anopheles and Culex by the resting position of the larvae which was further confirmed at the emergence of nulliparous using morphological keys; length of maxillary palp, wing spot, mouthpart, and abdominal end.

\subsection{Morphological Identification of Wild Caught Mosquito} Vectors. All the mosquito samples collected were identified by female morphology and male genitalia. Genus level identifications were made with multiple approaches using a morphology key which includes length of maxillary palp, wing spot, mouthpart, and abdominal end at 40× magnification [19].

2.4.1. Extraction of DNA. DNA was extracted using the TRIzol ${ }^{\circledR}$ reagent [20]. The mosquitoes parts (the head, thorax, and abdomen) were homogenized in the TRIzol ${ }^{\circledR}$ reagent using a glass Teflon at $25^{\circ} \mathrm{C}$ and vortexed to ensure thorough and complete isolation and solubilisation of the macromolecules. The resulting mixture was then separated into three phases (a lower red phenol-chloroform phase, an interphase, and a colourless upper aqueous phase). $250 \mu \mathrm{l}$ back extraction buffer (4 M guanidine thiocyanate; $50 \mathrm{mM}$ sodium citrate; $1 \mathrm{M}$ Tris, $\mathrm{pH}$ 8.0) was added to the phenol phase and the interphase, and the mixtures were incubated 
at room temperature for $10 \mathrm{~min}$. Samples were then centrifuged at $13200 \mathrm{rpms}$ for 15 minutes at $4^{\circ} \mathrm{C}$. The upper phase was removed and an equal volume of $100 \%$ isopropanol was added and incubated overnight at $-80^{\circ} \mathrm{C}$. After incubation, samples were centrifuged at $13,200 \mathrm{rpm}$ for 15 minutes at $4^{\circ} \mathrm{C}$. The supernatant was removed, and the pellets were washed 3 times with $70 \%$ ethanol and then eluted with the TAE buffer in a final volume of $20 \mu \mathrm{l}$ at pH 8.0 and the purity was determined at A260/280 [21, 22].

2.4.2. Molecular Identification of Wuchereria bancrofti. Wuchereria bancrofti collected from the wild caught infected mosquitoes were identified using the PCR technique. Primers (Table 1) were used to amplify the $188 \mathrm{bp}$ SspI repeats of Wuchereria bancrofti [23]. PCR was performed in a thermocycler (Applied Biosystems) with conditions set at $96^{\circ} \mathrm{C}$ for 15 seconds followed by 25 cycles of $94^{\circ} \mathrm{C}$ for 3 seconds, $56^{\circ} \mathrm{C}$ for 3 seconds, and $72^{\circ} \mathrm{C}$ for 5 seconds [24, 25]. A total reaction volume of $25 \mu \mathrm{l}$ containing $2 \mu \mathrm{l}$ of DNA sample, $10 \mathrm{mM}$ Tris- $\mathrm{HCl}$, pH 9.2, $2 \mathrm{mM} \mathrm{MgCl}_{2}, 75 \mathrm{mM} \mathrm{KCl}$, $1.2 \mathrm{mM}$ of each deoxynucleotide triphosphate, $10 \mathrm{pmol}$ each of SspI forward and reverse oligonucleotide primers (Table 1), and 2 unit of Taq polymerase was used.

(1) RNA Extraction. The clear aqueous surface obtained from the mosquito homogenate during DNA isolation was transferred into a fresh $1.5 \mathrm{~mL}$ RNase-free tube with equal volume of RNase-free $70 \%$. Ethanol was added after which the mixture was transferred into an RNeasy spin column and centrifuged at $18,000 \mathrm{rpm}$ for 30 seconds. Thereafter, the flow through was discarded. $700 \mu \mathrm{l}$ of the RW1 buffer was added to the column and centrifuged at $18,000 \mathrm{rpm}$ for 30 seconds and transferred to a new collection tube into which $500 \mu \mathrm{l}$ of RPE buffer was added and centrifuged for 2 minutes at maximum speed. The flow through was discarded, and the column was transferred to a $1.5 \mathrm{ml}$ RNasefree collection tube and eluted with $20 \mu \mathrm{l}$ RNase-free water. The concentration and purity of extracted RNA were determined at A260/A280.

(2) Complimentary DNA (cDNA) Synthesis. Reverse transcription of the RNA to cDNA was carried out with the SuperScript First-Strand Synthesis System. The procedure was based on Invitrogen's protocol (New England Bio Lab $_{\text {inc }}$ ) in a $20 \mu \mathrm{l}$ reverse transcription mixture. $1 \mu \mathrm{l}$ of the isolated RNA was mixed with $2 \mu \mathrm{l}$ of random hexamers, and nuclease-free water was added to make up the total volume. It was then incubated at $65^{\circ} \mathrm{C}$ for 5 minutes, and thereafter, ProtoScript II reaction mix (2x) and ProtoScript II enzyme mix (10x) were added and then incubated at $25^{\circ} \mathrm{C}$ for 5 minutes followed by $42^{\circ} \mathrm{C}$ for 1 hour. Finally, the enzyme reaction was inactivated at $80^{\circ} \mathrm{C}$ for 5 minutes.

(3) Amplification of Actin-1. Polymerase chain reaction was carried out in a thermal cycler (Applied Biosystems) with a total reaction volume of $50 \mu \mathrm{l}$ using a universal primer sequence (Table 1) for the amplification of actin-1 cDNA [13]. The PCR mix contained $5 \mu \mathrm{l} 10 \mathrm{X}$ PCR buffer, $1.5 \mu \mathrm{l} \mathrm{\textrm {Mgl } _ { 2 }}$
(50 mM), $1 \mu \mathrm{ldNTP}(10 \mathrm{mM}), 1 \mu \mathrm{l}$ of each primer $(10 \mathrm{mM})$, $0.4 \mu \mathrm{l}$ Taq polymerase $(5 \mathrm{U} / \mu \mathrm{l}), 2 \mu \mathrm{l} \mathrm{cDNA}$, and $38.1 \mu \mathrm{l}$ of DEPC-treated water with conditions set at $96^{\circ} \mathrm{C}$ for 15 seconds followed by 25 cycles of $94^{\circ} \mathrm{C}$ for 3 seconds, $56^{\circ} \mathrm{C}$ for 3 seconds, and $72^{\circ} \mathrm{C}$ for 5 seconds [25].

(4) Agarose Gel Electrophoresis. The obtained PCR products from identification of Wuchereria bancrofti and actin- 1 were visualized on prepared $1.5 \%$ agarose gel stained with $0.5 \mu \mathrm{g} /$ $\mathrm{ml}$ ethidium bromide to detect the amplified DNA fragments. Accordingly, $5 \mu \mathrm{L}$ of each PCR product was added to $1 \mu \mathrm{L}$ of the loading dye for electrophoresis. The gel was prepared by weighing $1.5 \mathrm{~g}$ of agarose powder and dissolved in $100 \mathrm{ml}$ of TAE buffer and poured on a casting tray to allow polymerization and solidification at room temperature and then electrophoresis was performed using $1 \mathrm{x}$ TAE in a minigel system at 100 volts for one hour. The gel was visualized on a UV transilluminator where the sizes of the PCR products were estimated by comparison with the mobility of a 100 bp DNA molecular ladder [26].

(5) Sequencing of Actin-1 Gene Amplicon. The PCR products were purified using a QIAQUICK PCR Purification Kit (QIAGEN, USA) following the manufacturer's protocol. Sequencing was carried out using the BigDye Terminator v3.1 Cycle Sequencing Kit on purified PCR products in a DNA thermal cycler.

(6) Quantitative Polymerase Chain Reaction ( $q P C R$ ). Evaluation of mRNA levels was done using the SYBRGreenER ${ }^{\mathrm{TM}}$ qPCR SuperMix Universal (Thermo Fisher Scientific, USA) following the manufacturer's instructions, with a total reaction volume of $20 \mu \mathrm{l}$. Primers (Table 1) for both actin-1 and GAPDH (internal control) were equally used in triplicates. For the reaction, $2 \mu \mathrm{l}$ of reverse-transcribed products (cDNA), $10 \mu \mathrm{l}$ Luna universal qPCR mix, $1 \mu \mathrm{l}$ of RNAse-free water, and $1 \mu \mathrm{l}$ each of forward and reverse primers were present in a $20 \mu \mathrm{l}$ final volume. Thermo cycler conditions consisted of 1 cycle $-95^{\circ} \mathrm{C}$ for 15 minutes and forty cycles $95^{\circ} \mathrm{C}$ for 15 seconds, $62^{\circ} \mathrm{C}$ for 15 seconds, and $72^{\circ} \mathrm{C}$ for 30 seconds. A melting curve was obtained for each quantitative PCR run, and the second derivative maximum method was used to determine the crossing point $(\mathrm{Cp})$ for individual samples [27]. The real-time PCR data generated (Table 2) were analyzed using the $2^{-\Delta \Delta C T}$ relative quantization method [28].

2.5. Data Analysis. To address biological variability, experiments were repeated at least three (3) times and appropriate data were presented as mean \pm SEM. Variability between groups was measured using the $t$-test with the aid of Statistical Package for Social Sciences Software version 20.0. Level of significance measured at $P<0.05$ was considered statistically significant using least significance difference (LSD).

\section{Results}

3.1. Mosquito Vector Distribution and Morphological Identification. Table 3 shows that 100 larvae were collected 
TABLE 1: List of primers used for conventional polymerase chain reaction (PCR) and relative quantitative real-time polymerase chain reaction (qPCR).

\begin{tabular}{|c|c|c|c|}
\hline Genes & Amplicon size (bp) & Forward $5^{\prime} \longrightarrow 3^{\prime}$ & Reverse $5^{\prime} \longrightarrow 3^{\prime}$ \\
\hline SspI & 188 & CGTGATGGCATCAAAGTAGGG & СССТСАСТTACСATAAGACAAC \\
\hline Actin-1 (PCR) & 683 & TGGTCGGCATGGGTCAGAAGGACTC & GATTCCATACCCAGGAAGGATGG \\
\hline Actin-1 (qPCR) & 154 & GCACGGTATCATCACCAACTG & CATGATCTGGGTCATCTTCTCG \\
\hline GAPDH & 194 & ACAGACGCTAGTTATCAACGTA & ACCGTGGGTCGAATCGTA \\
\hline
\end{tabular}

TABle 2: Cycle threshold (Ct) values and calculations for relative fold change in the expression of the actin-1 gene.

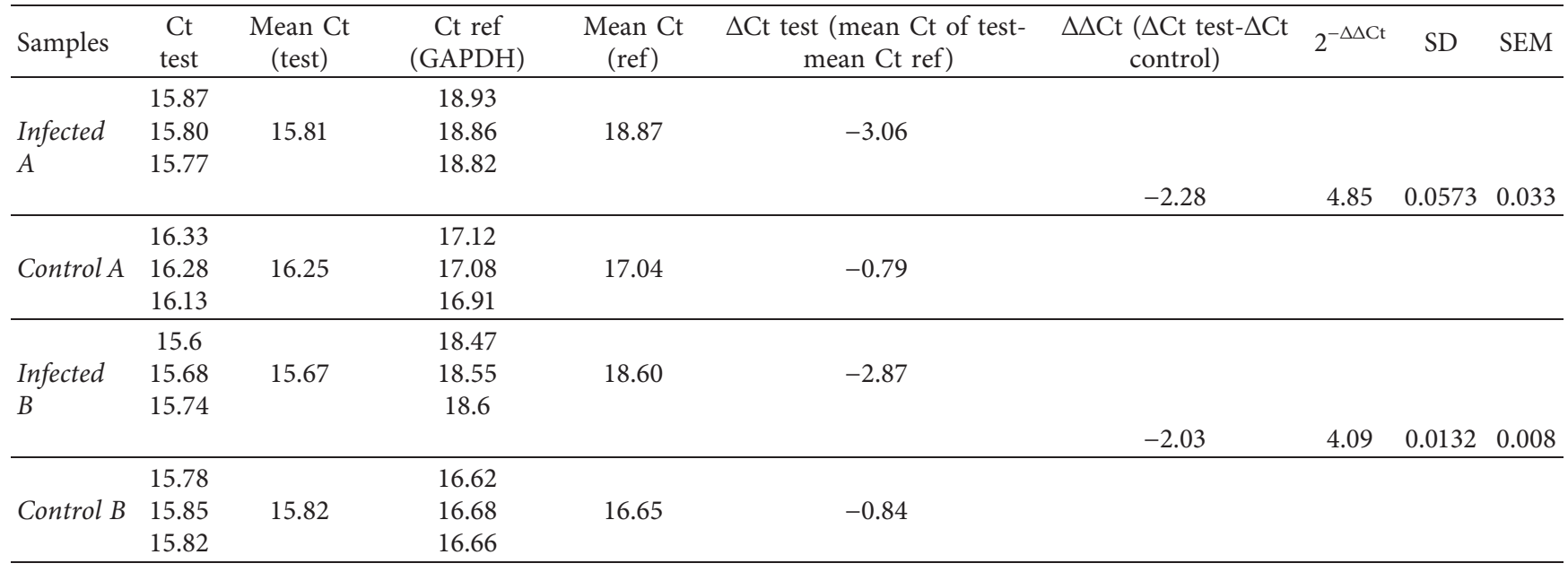

A: Anopheles gambiae and B: Culex quinquefasciatus.

TABle 3: Distribution of mosquito vectors and the infection rate of Wuchereria bancrofti.

\begin{tabular}{lcccc}
\hline & Total samples & Infected mosquitoes & \multicolumn{2}{c}{ Mosquito types } \\
& & & Culex quinquefasciatus & Anopheles gambiae \\
\hline Wild caught & 2000 & $2(0.002 \%)$ & $924(46.2 \%)$ & $783(39.2 \%)$ \\
Laboratory bred & 100 & - & $41(82.0 \%)$ & $33(66.0 \%)$ \\
\hline
\end{tabular}

from breeding sites in the study area and reared in the laboratory to adult, of which 50 were Culex and 50 Anopheles as identified from the larvae stage; however, only 41 and 33 emerged from Culex and Anopheles, respectively, due to mortality. Out of the 2000 wild caught mosquitoes, 784 were Anopheles gambiae sl and 925 were identified to be Culex quinquefasciatus of which one from each group was found to be positive with $W$. bancrofti. The remaining 291 wild caught mosquitoes were identified to be Aedes aegypti and not infected with $W$. bancroft $i$. The morphological features used for the identification included length of maxillary palps, wing pale spots, and scale patterns on the abdomen as previously described [29].

3.2. Molecular Detection of Wuchereria bancrofti and Amplification of Actin-1. The filarial parasite from this research was identified by polymerase chain reaction using SspI primers [23] with an amplicon size of $188 \mathrm{bp}$ as shown in Figure 1. Figure 2 shows the amplicon of actin- 1 gene amplified using an actin-1 universal primer. The bands from gel were excised (Figure 2), purified, and sequenced (Figures 3(a)-3(d)).

3.3. Expression of Actin-1. Figure 4 shows the result of actin1 expression in wild caught Wuchereria bancrofti-infected
Anopheles gambiae sl and Culex quinquefasciatus. The expression fold of the actin-1 gene in Anopheles gambiae was increased by 4.85 and that of Culex quinquefasciatus showed a fold increase of 4.09 relative to controls. The proportional fold increase of actin-1 gene expression was found to be higher in Anopheles gambiae sl by 0.7526 when compared with Culex quinquefasciatus although not statistically significant $(P>0.05)$.

\section{Discussion}

Wuchereria bancrofti is the major cause of lymphatic filariasis transmitted by mosquito vectors. In the vector-parasite interaction and among other proteins, actin-1 has been implicated for successful transmission of the pathogen. Actin-1 is an extracellular pathogen recognition factor that mediates antibacterial defense [12]. Insect actin- 1 is secreted from cells upon immune challenge through an exosomeindependent pathway, and it binds to the surfaces of bacteria, mediating their phagocytosis and direct killing. Globular and filamentous actins display distinct functions as extracellular immune factors [30]. Unfortunately, in an endemic country like Nigeria, there has been little or no attention given to the pathogen-vector interactions in research studies to decipher the biological factors involved. In 


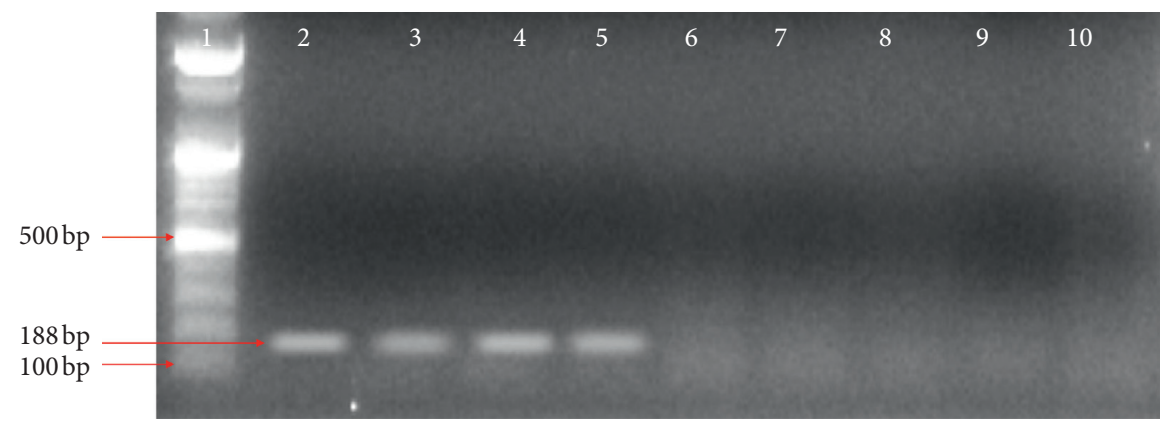

Figure 1: Agarose gel electrophoresis to detect SspI Wuchereria bancrofti. Lane $1=100 \mathrm{bp}$ molecular marker; Lane 2 and $3=$ Anopheles (infected with Wuchereria bancrofti); Lane 4 and $5=$ Culex (infected with Wuchereria bancrofti); Lane 6 and $7=$ Anopheles (negative); Lane 8 and $9=$ Culex (negative).

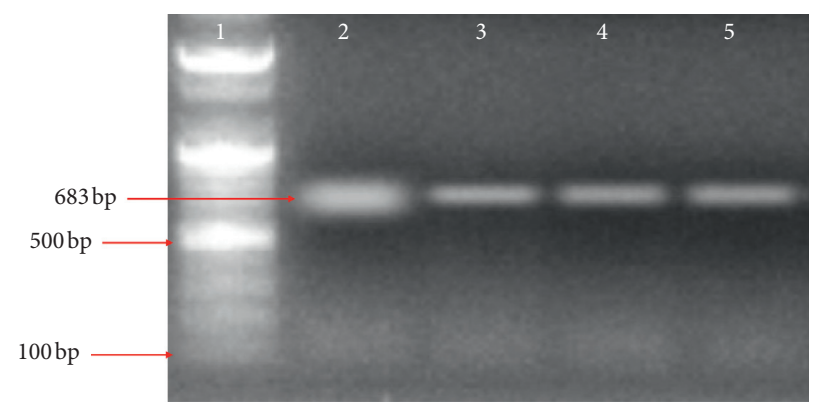

FIGURE 2: Agarose gel electrophoresis to amplify actin-1 in both infected and control mosquitoes. Lane $1=100$ bp molecular marker, Lane 2 and 3 = infected mosquito (Anopheles and Culex, respectively), and Lane 4 and $5=$ control (Anopheles and Culex, respectively).

this communication, we identified and evaluated actin-1 gene expression in wild caught Wuchereria bancrofti-infected mosquito vectors obtained from their natural environment during an epidemiological study in Tsafe Local Government Area of Zamfara State, Nigeria.

From this study, only 2 mosquitoes were found to be infected with a filarial nematode from which one was Anopheles gambiae sl and the other was Culex quinquefasciatus. The filarial specimen was Wuchereria bancrofti confirmed by PCR amplification of the SspI gene for the detection of Wuchereria bancrofti with a corresponding amplicon size of $188 \mathrm{bp}$. This results corroborate with the result which tested the hypothesis that Wuchereria bancrofti can be identified in mosquito vectors using the SspI repeats [24]. Similarly, a survey of bancroftian filariasis infection in humans and Culex mosquitoes in the western Brazilian Amazon region showed that implications for transmission and control using SspI repeats as a signature to the parasite have proven to be a powerful tool to evaluate the contamination intensity of the mosquitoes in endemic areas. Due to these advantages, it has replaced the conventional dissection methods for the diagnosis [31]. Similarly, the SspI repeat has a unique recognition site for restriction endonuclease in all or most of the repeat copies. This DNA family is dispersed, genus-specific, and exists in all of the different geographic isolates of Wuchereria bancrofti tested [23].

The expression fold increase of actin-1 in Anopheles gambiae was found to be 4.85 and 4.09 for Culex quinquefasciatus as compared to the controls. These values indicate that the expression of actin-1 is upregulated in Culex quinquefasciatus which agrees with other reported upregulation of actin-1 protein upon infection and development of the filarial parasites within their vectors [12]. Actin-1 expression in Anopheles gambiae was 0.7526 more than that of Culex quinquefasciatus which may be as a result of the fact that Anopheles species are the predominant vectors in the study area and they have adapted to ameliorating the effect of parasite infection via various mechanisms which include melanisation, encapsulation, and phagocytosis. Mosquito immune machinery involves biochemical reactions using exoskeleton cuticle, epidermis physical and chemical properties, gut epithelium, and reproductive accessory glands [32]. The major feature in this immune machinery is the recognition of nonself-parts via pattern recognition receptors (PRRs) that identify pathogen-associated molecular patterns (PAMPs) which trigger the activation of cellular and humoral immune responses, both acting together to eliminate pathogens by surrounding of pathogens using melanin [33]. Typically, cellular responses include phagocytosis, nodulation, and encapsulation, while humoral include antimicrobial systemic molecules, nitric oxide (NO) production, and lysozymes [14]. Mosquitoes also possess innate immunity to combat pathogen infection [11], supporting the facts that actin-1 is indeed part of the innate immune response as a consequence of parasite-vector interactions [12]. The innate immune system includes phagocytic cells in the circulation and tissues. These cells recognize and engulf pathogens through germline-encoded pattern recognition receptors specific for microbial products such as the toll-like receptors [34]. The toll pathway in mosquitoes has been found to 


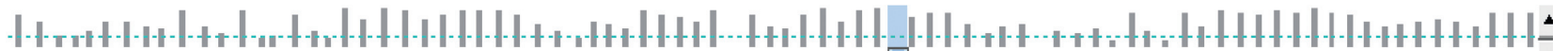

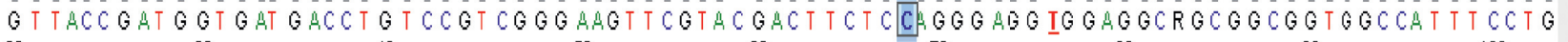

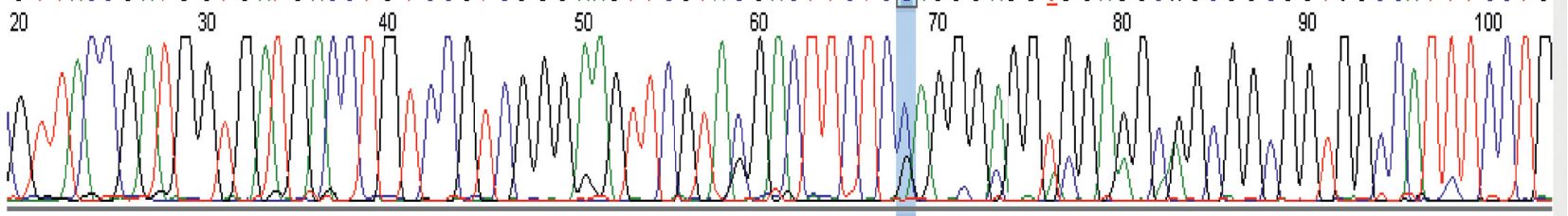

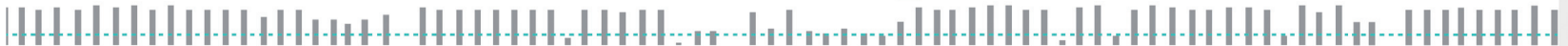

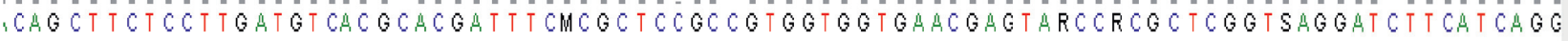
(30) H-H.H.

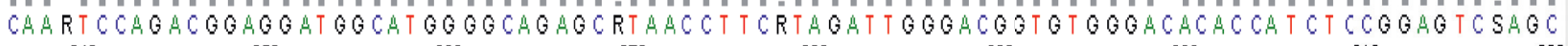
240 250 260 280 290

300

310

320

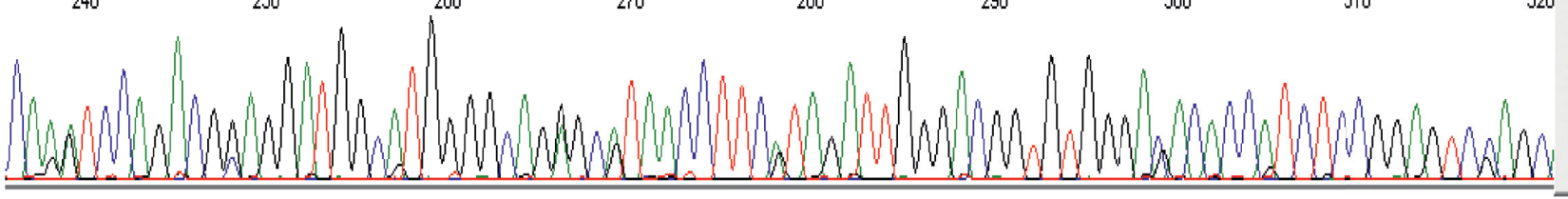
H.t.

(a)

$\therefore$ GCTCGTTACCGATGGTGATGACCTGTCCGTCGGGAAGTCGTACGACTTCTCCAGGGAGGTGGAGGCGGCGGCGGTGGCCATTTCO (n) ; TAGCACAGCTTCTCCTTGATGTCACGSACGATTTCMCBCTCRGCCGTGGTGGTGAACGATARCORCOCTCGGTSAGGATCTTCATCH 130

140

150

150

170

180

190

200

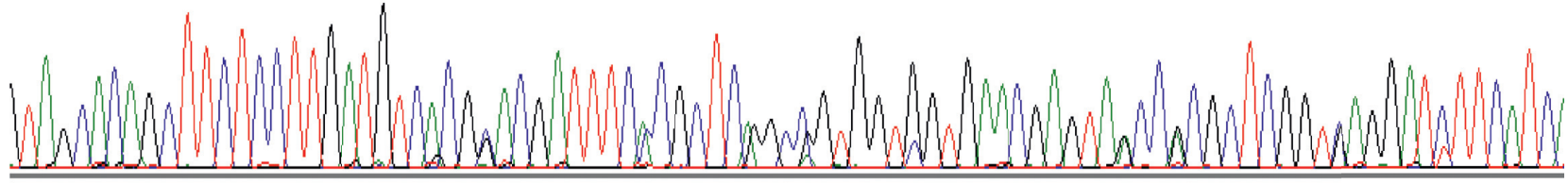

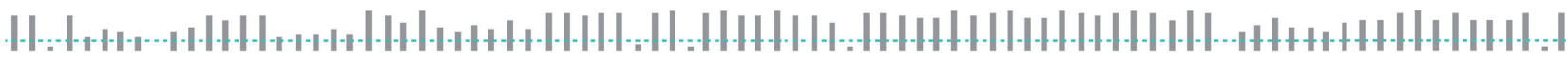
CCAGCCA _ TCCAGACGGAGGATGGCATGGGGCAGGGC_TACCTTC_TAGATTGGGACGGTGTGGOA_ACACCATCTCCOGAGTCSA ] 250 260 270 280 $29]$ 

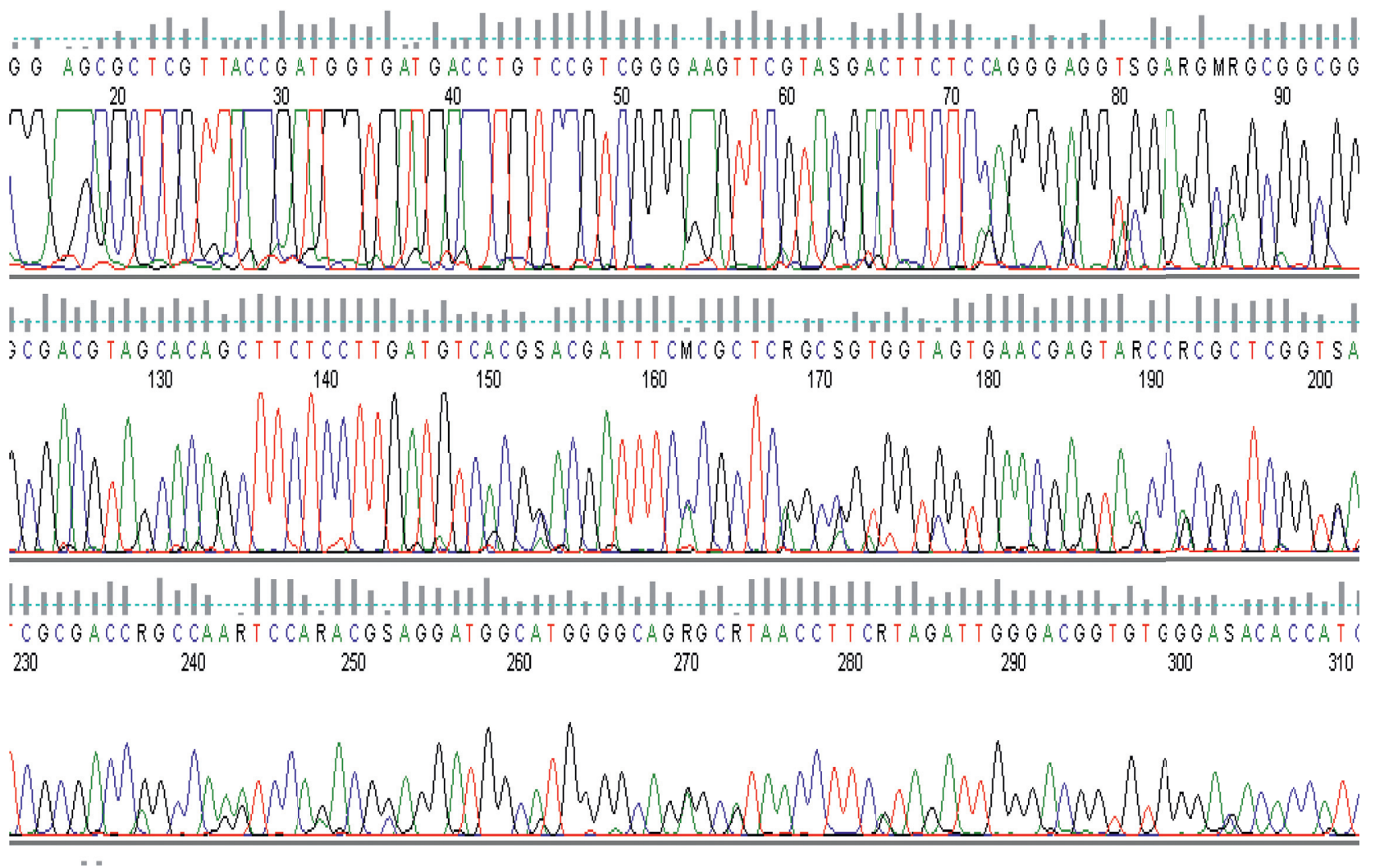

(c)

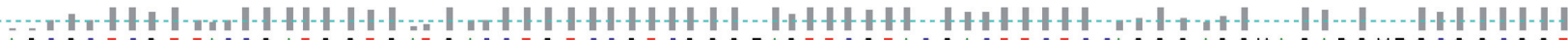
AGCGCTCGTTACCGATGGTGATGACCTGTCCGTCGQGRAGT TCGTACGACT TCTCCAGGGAGGMAGARGMRGCGGCGGT

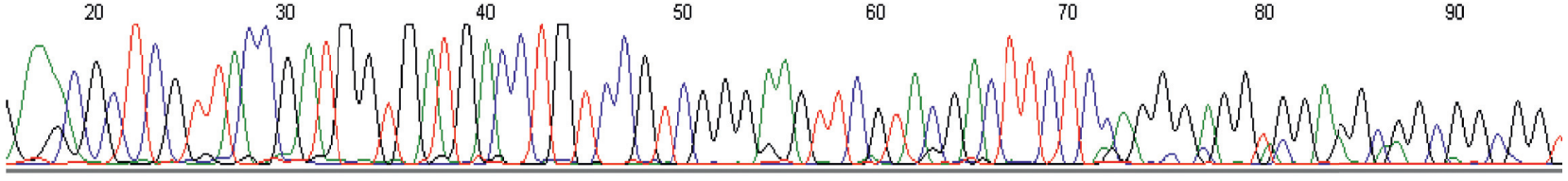

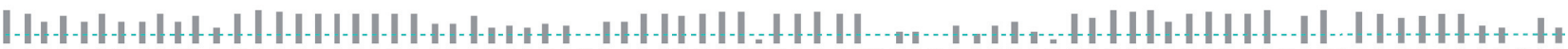
$3 A C G T A G C A C A G C T T C T C C T T Q A T G T C A C G S A C G A T T C M C G C T C R G C N G T Q G T S G T G A A C G A G T A R C C R C G C T C G G T S A G$

140

160

180

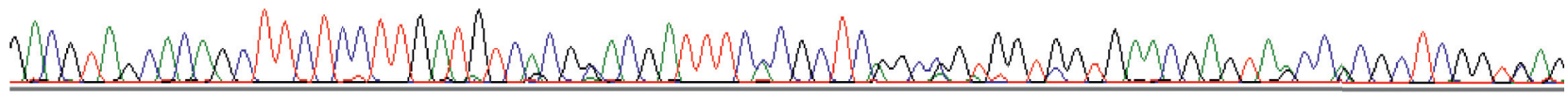

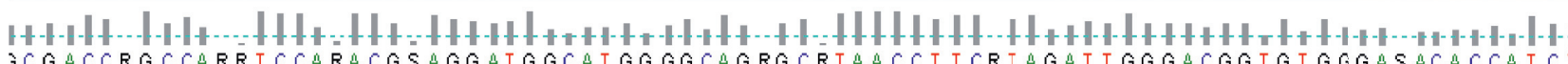
3CGACCRGCCART CCARACGSAGGATGGCATGG GGCAGRGCRTAACCT TCRTAGATTGGGACGGTGTGGGASACACCATC 310

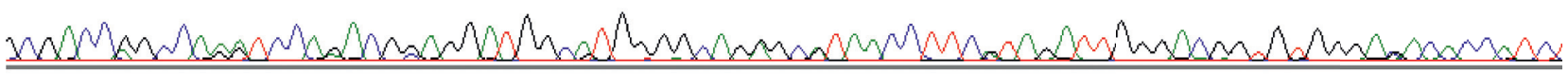

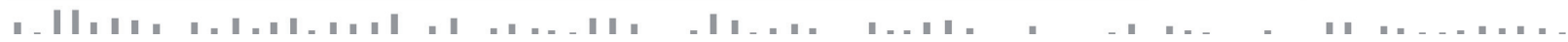

(d)

Figure 3: (a) Sequence chromatograms of actin-1 with a region used for primer design from infected Anopheles gambiae sl. (b) Sequence chromatograms of actin-1 with a region used for primer design from control Anopheles gambiae sl. (c) Sequence chromatograms of actin-1 with a region used for primer design from infected Culex quinquefasciatus. (d) Sequence chromatograms of actin-1 with a region used for primer design from control Culex quinquefasciatus. 


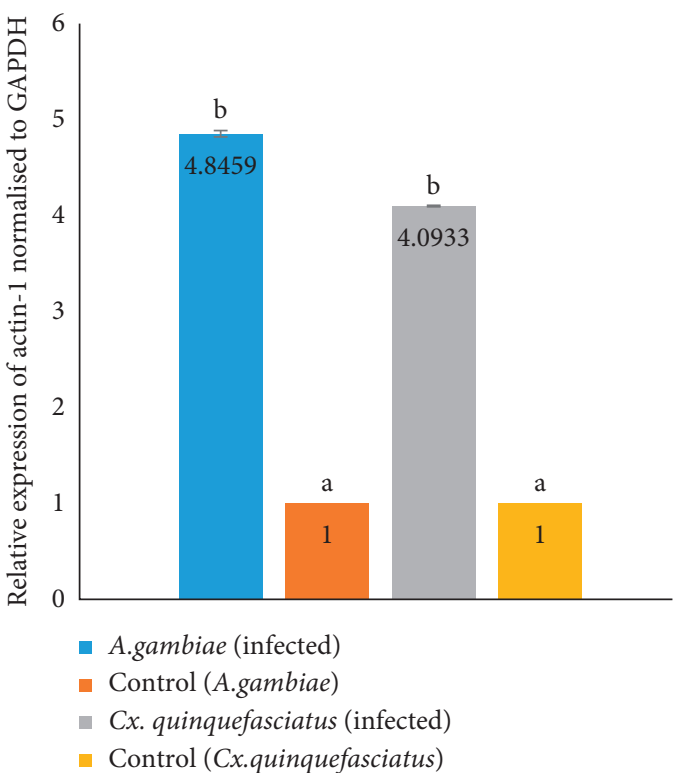

FIgURE 4: Expression of actin-1 in infected Anopheles gambiae and Culex quinquefasciatus. Bars (means) with different superscript are statistically significant at $\mathrm{p}<0.05$.

control hemocyte proliferation and plays a critical role in cellular immune response, for the expression of actin-1, transferrin, and defensin proteins critical to encapsulation and killing of parasites [35].

\section{Conclusion}

Wild caught Anopheles gambiae sl and Culex quinquefasciatus infected with $W$. bancrofti from Tsafe Local Government Area of Zamfara State Nigeria showed upregulation of the actin-1 gene. Therefore, targeting actin- 1 as one of the protein that is upregulated due to interaction between the parasite and the vector could serve as a new strategy for the control of the parasite transmission in endemic areas.

\section{Data Availability}

The raw data and samples used to support the findings of this study are available from the corresponding author upon request.

\section{Conflicts of Interest}

The authors declare that they have no conflicts of interest.

\section{Authors' Contributions}

Moses Edache Entonu, Aliyu Muhammad, and Iliya S. Ndams conceptualized, executed, and interpreted the study and were also involved in writing and proof reading. Aliyu Muhammad and Iliya S. Ndams designed the study.

\section{Acknowledgments}

The authors appreciate the assistance of Dr. Muawiya Abarshi Musa of the Department of Biochemistry, and Aliyu
Abdulwahab and Agada Jeremiah of the Department of Zoology, Ahmadu Bello University, Zaria. This research was funded by the Africa Centre of Excellence for Neglected Tropical Disease and Forensic Biotechnology, Ahmadu Bello University, Zaria, to Entonu Moses Edache.

\section{References}

[1] L. M. Gedge, A. A. Bettis, M. H. Bradley, T. D. Hollingsworth, and H. C. Turner, "Economic evaluations of lymphatic filariasis interventions: a systematic review and research needs," Parasites and Vectors, vol. 11, no. 1, pp. 1-18, 2018.

[2] T. B. Nutman, "Insights into the pathogenesis of disease in human lymphatic filariasis," Lymphatic Research and Biology, vol. 11, no. 3, pp. 144-148, 2013.

[3] M. Dogara, H. Nock, R. Agbede, S. Ndams, and K. Joseph, "Prevalence of lymphatic filariasis in three villages in Kano State, Nigeria," The Internet Journal of Tropical Medicine, vol. 8, no. 1, pp. 1-18, 2012.

[4] S. O. Elkanah, T. C. Swemwua, D. S. Elkanah et al., "Status of lymphatic filariasis in five communities of Yorro local government area, Taraba state, Nigeria," Nigerian Journal of Parasitology, vol. 39, no. 1, p. 42, 2018.

[5] D. M. Steiger, P. Johnson, D. W. Hilbert, S. Ritchie, D. Jones, and S. G. W. Laurance, "Effects of landscape disturbance on mosquito community composition in tropical Australia," Journal of Vector Ecology, vol. 37, no. 1, pp. 69-76, 2012.

[6] D. Famakinde, "Mosquitoes and the lymphatic filarial parasites: research trends and budding roadmaps to future disease eradication," Tropical Medicine and Infectious Disease, vol. 3, no. 1, p. 4, 2018.

[7] J. F. Hillyer, "Mosquito immunity," Advances in Experimental Medicine and Biology, vol. 708, pp. 218-238, 2010.

[8] K. P. Paily, S. L. Hoti, and P. K. Das, "A review of the complexity of biology of lymphatic filarial parasites," Journal of Parasitic Diseases, vol. 33, no. 1-2, pp. 3-12, 2009.

[9] E. B. Beckett and B. Boothroyd, "Mode of nutrition of the larvae of the filarial nematode Brugia pahangi," Parasitology, vol. 60, no. 1, pp. 21-26, 1970.

[10] M. J. Lehane and B. R. Laurence, "Flight muscle ultrastructure of susceptible and refractory mosquitoes parasitized by larval brugia pahangi," Parasitology, vol. 74, no. 1, pp. 87-92, 1977.

[11] W. S. Lee, J. A. Webster, E. T. Madzokere, E. B. Stephenson, and L. J. Herrero, "Mosquito antiviral defense mechanisms: a delicate balance between innate immunity and persistent viral infection," Parasites and Vectors, vol. 12, no. 1, pp. 1-12, 2019.

[12] B. Agiesh Kumar and K. P. Paily, "Actin protein up-regulated upon infection and development of the filarial parasite, Wuchereria bancrofti (Spirurida: Onchocercidae), in the vector mosquito, Culex quinquefasciatus (Diptera: Culicidae)," Experimental Parasitology, vol. 118, no. 3, pp. 297-302, 2008.

[13] M. Staley, K. S. Dorman, L. C. Bartholomay et al., "Universal primers for the amplification and sequence analysis of actin-1 from diverse mosquito species," Journal of the American Mosquito Control Association, vol. 26, no. 2, pp. 214-218, 2010.

[14] A. L. Flores-Villegas, P. M. Salazar-Schettino, A. CórdobaAguilar et al., "Immune defence mechanisms of triatomines against bacteria, viruses, fungi and parasites," Bulletin of Entomological Research, vol. 105, no. 5, pp. 523-532, 2015.

[15] S. K. Sreenivasamurthy, G. Dey, M. Ramu et al., "A compendium of molecules involved in vector-pathogen interactions pertaining to malaria," Malaria Journal, vol. 12, no. 1, p. 216, 2013. 
[16] M. Janeh, D. Osman, and Z. Kambris, "Damage-induced cell regeneration in the midgut of Aedes albopictus mosquitoes," Scientific Reports, vol. 7, no. 1, pp. 1-10, 2017.

[17] G. S. Abdulwahab Aliyu and I. S. Ndams, "Studies on some aspect of lymphatic filariasis in Tsafe and talatan-mafara local government area, Zamfara state, Nigeria," Ahmadu Bello Univesity, pp. 23-27, School of Postgraduate Studies Publication, Zaria, Nigeria, 2016.

[18] J. C. Anosike, B. E. Nwoke, C. O. Onwuliri et al., "Prevalence of parasitic diseases among nomadic Fulanis of south-eastern Nigeria," The Annals of Agricultural and Environmental Medicine, vol. 11, no. 2, pp. 221-225, 2004.

[19] Y. M. Huang and L. M. Rueda, "Pictorial keys to the sections, groups, and species of the Aedes (Finlaya) in the Afrotropical region (Diptera: Culicidae)," Zootaxa, vol. 4221, no. 1, pp. 131-141, 2017.

[20] P. Chomczynski and N. Sacchi, "Single-step method of RNA isolation by acid guanidinium thiocyanate-phenol-chloroform extraction," Analytical Biochemistry, vol. 162, no. 1, pp. 156-159, 1987.

[21] D. A. Triant and A. Whitehead, "Simultaneous extraction of high-quality RNA and DNA from small tissue samples," Journal of Heredity, vol. 100, no. 2, pp. 246-250, 2009.

[22] J.-J. Qin, Z.-Y. Lu, Z.-P. Jiao, X.-J. Zhu, Y.-X. Wang, and H. Tang, "Modified TRIzol method for RNA and DNA coextraction from blood," Fa Yi Xue Za Zhi, vol. 29, no. 3, pp. 209-211, 2013.

[23] M. Zhong, T. B. Nutman, E. A. Ottesen et al., "A polymerase chain reaction assay for detection of the parasite Wuchereria bancrofti in human blood samples," The American Journal of Tropical Medicine and Hygiene, vol. 54, no. 4, pp. 357-363, 1996.

[24] R. L. Korte, G. Fontes, J. D. S. A. A. Camargo et al., "Survey of bancroftian filariasis infection in humans and culex mosquitoes in the western Brazilian Amazon region: implications for transmission and control," Revista da Sociedade Brasileira de Medicina Tropical, vol. 46, no. 2, pp. 214-220, 2013.

[25] C. Bouakaze, J. Eschbach, E. Fouquerel et al., "OpenLAB": a 2hour PCR-based practical for high school students," Biochemistry and Molecular Biology Education, vol. 38, no. 5, pp. 296-302, 2010.

[26] P. Y. Lee, J. Costumbrado, C. Y. Hsu, and Y. H. Kim, “Agarose gel electrophoresis for the separation of DNA fragments," Journal of Visualized Experiments, vol. 20, no. 62, p. 3923, 2012.

[27] A. Muhammad, A. Funmilola, I. A. Aimola, I. S. Ndams, M. H. Inuwa, and A. J. Nok, "Kolaviron shows anti-proliferative effect and down regulation of vascular endothelial growth factor-C and toll like receptor-2 in Wuchereria bancrofti infected blood lymphocytes," Journal of Infection and Public Health, vol. 10, no. 5, pp. 661-666, 2017.

[28] K. J. Livak and T. D. Schmittgen, "Analysis of relative gene expression data using real-time quantitative PCR and the $2^{-\Delta \Delta \mathrm{CT}}$ method," Methods, vol. 25, no. 4, pp. 402-408, 2001.

[29] B. T. Lamidi, E. B. Alo, and R. S. Naphtali, "Mosquito species diversity and distribution in three riverine communities in taraba state, north-eastern Nigeria," IOSR Journal of Pharmacy and Biological Sciences, vol. 12, no. 2, pp. 21-28, 2017.

[30] S. L. Sandiford, Y. Dong, A. Pike, B. J. Blumberg, A. C. Bahia, and G. Dimopoulos, "Cytoplasmic actin is an extracellular insect immune factor which is secreted upon immune challenge and mediates phagocytosis and direct killing of bacteria, and is a Plasmodium antagonist," PLoS Pathogens, vol. 11, no. 2, Article ID e1004631, 2015.
[31] M. J. Bockarie, E. M. Pedersen, G. B. White, and E. Michael, "Role of vector control in the global program to eliminate lymphatic filariasis," Annual Review of Entomology, vol. 54, no. 1, pp. 469-487, 2009.

[32] J. P. Gillespie, M. R. Kanost, and T. Trenczek, "Biological mediators of insect immunity," Annual Review of Entomology, vol. 42, no. 1, pp. 611-643, 1997.

[33] I. González-Santoyo and A. Córdoba-Aguilar, "Phenoloxidase: a key component of the insect immune system," Entomologia Experimentalis et Applicata, vol. 142, no. 1, pp. 1-16, 2012.

[34] N. Ismail, J. P. Olano, H.-M. Feng, and D. H. Walker, "Current status of immune mechanisms of killing of intracellular microorganims," FEMS Microbiology Letters, vol. 207, no. 2, pp. 111-120, 2002.

[35] S. Valanne, J.-H. Wang, and M. Rämet, "The Drosophila toll signaling pathway," The Journal of Immunology, vol. 186, no. 2, pp. 649-656, 2011. 